\title{
Multiple factors are required for poly(A) addition to a mRNA $3^{\prime}$ end
}

\author{
Michael A. McDevitt, ${ }^{1}$ Gregory M. Gilmartin, ${ }^{1,3}$ Westley H. Reeves, ${ }^{2}$ and Joseph R. Nevins ${ }^{1,3}$ \\ ${ }^{1}$ Howard Hughes Medical Institute, Laboratory of Molecular Cell Biology, The Rockefeller University, New York, New York \\ 10021 USA; ${ }^{2}$ Laboratory of Immunology, The Rockefeller University, New York, New York 10021 USA
}

Polyadenylation of pre-mRNAs in the nucleus involves a specific endonucleolytic cleavage, followed by the addition of $\sim 200$ adenylic acid residues. We have assayed HeLa nuclear extracts for the activity that catalyzes the $\operatorname{poly}(\mathrm{A})$ addition reaction. The authenticity of the in vitro assay was indicated by the observation that the poly(A) addition reaction requires an AAUAAA element near the $3^{\prime}$ terminus of the substrate and that the poly(A) tract added in vitro is $\sim 200$ nucleotides in length. We have fractionated nuclear extracts in order to define components involved in specific poly(A) addition. No single fraction from DEAE-Sephacel chromatography of a HeLa nuclear extract possessed the specific poly(A) addition activity. However, if the various fractions were recombined, activity was restored, indicating the presence of multiple components. Further fractionation revealed the presence of at least two factors necessary for the poly(A) addition reaction. The reconstituted system retains the characteristics and specificity seen in the crude extract. Additional purification of one of the factors strongly suggests it to be a previously characterized poly(A) polymerase which, when assayed in the absence of the other factor, can add AMP to an RNA terminus but without specificity. Thus, the other component of the reaction may provide specificity to the process. In contrast to the $3^{\prime}$ cleavage reaction, the poly(A) addition machinery does not possess an essential RNA component, as assayed by micrococcal nuclease digestion, nor do anti-Sm sera inhibit the reaction. Thus, the total process of formation of a polyadenylated mRNA $3^{\prime}$ end is complex and requires the concerted action of distinct nuclear components.

[Key Words: poly(A) addition activity; poly(A) polymerase; nuclear components; mRNA 3' end formation]

Received September 4, 1987; revised version accepted March 30, 1988.

As detailed in Gilmartin et al. (this issue), the formation of a polyadenylated mRNA involves a specific endonucleolytic cleavage at the mRNA 3 ' terminus and the subsequent polymerization of $\sim 200$ adenylic acid residues onto the cleaved terminus (Nevins 1983; Birnstiel et al. 1985). Several lines of evidence suggest that these two events (cleavage and polyadenylation) are coupled and may involve a multicomponent complex. First, there is no indication, from either in vivo (Nevins and Darnell 1978; Manley et al. 1982), or in vitro studies, for a lag between cleavage and polyadenylation; a cleaved but nonpolyadenylated transcript has never been observed. Second, one of the sequence elements required for $3^{\prime}$-end cleavage, the AAUAAA, is also required for in vitro polyadenylation (Manley et al. 1985; Zarkower et al. 1986). Indirect RNase protection experiments in vitro suggest the formation of a complex involving the AAUAAA, and competition experiments suggest the possibility that the same complex is involved in both cleavage and polyadenylation (Zarkower and Wickens 1987). Polyadenylation-specific complexes have been identified in vitro, using nondenaturing, low percentage polyacrylamide gels (Skolnik-David et al. 1987; Zhang

${ }^{3}$ Present address: Howard Hughes Medical Institute, Department of $\mathbf{M i}$ crobiology and Immunology, Duke University Medical Center, POB 3054, Durham, North Carolina 27710 USA. and Cole 1987). One might expect that activities that mediate 3 '-end selection, cleavage of the RNA, and subsequent poly(A) addition are part of a multicomponent complex that functions within the cell.

Numerous previous studies have reported the identification and purification of activities from various sources that could polymerize AMP on the $3^{\prime}$ terminus of an RNA substrate (for review, see Edmonds and Winters 1976). In most cases, however, data have been lacking to demonstrate that the purified activities identified in extracts are equivalent to the activity that adds poly $(A)$ to the mRNA precursor. Only relatively recently has it been demonstrated that extracts are capable of an apparently authentic poly(A) addition reaction (Manley 1983). The complexity of this activity or its relationship to previously purified poly $(\mathrm{A})$ polymerases is not clear. Gilmartin et al. have demonstrated the existence of multiple factors required for the endonuclease-mediated cleavage of a pre-mRNA to generate the adenovirus L3 poly(A) addition site (this issue). In this paper, we describe the analysis of HeLa cell nuclear extracts and fractions for the ability to carry out the subsequent poly(A) addition reaction. Our criteria for the authenticity of the activity are twofold: first, that there is a requirement for the AAUAAA element and, second, that the length of the poly(A) tail which is added in vitro, approximates the in vivo 200-nucleotide poly(A) seg- 
ment. We find that, as with the cleavage reaction, there are multiple factors necessary for proper polyadenylation.

\section{Results}

\section{Properties and specificity of $\operatorname{poly}(A)$ addition}

Our assay for poly(A) addition is similar to that described previously by Manley (1983) and involves incubation of a labeled SP6 transcript with nuclear extracts in the presence of ATP and magnesium, followed by the analysis of the products by gel electrophoresis. As shown in Figure 1, SP6 RNAs containing the adenovirus type 5 (Ad5) L3 poly(A) site, the early SV40 poly(A) site, and the Ad5 E2A poly(A) site were polyadenylated efficiently, as evidenced by the slower mobility of the labeled RNA in the gel. In each case, the shifted RNA was approximately 100-300 nucleotides longer than the substrate, as estimated by comparison to various marker RNA species. Fractionation of the products by oligo(dT)-cellulose chromatography indicated that the altered mobility was indeed due to the addition of poly(A) (data not shown). Also, as shown in Figure 1, poly(A) addition appears to require an authentic poly(A) site. SP6 transcripts that do not contain a poly(A) site (SP64 and Geml) were not polyadenylated. Finally, as shown in Figure 1B, there was also efficient polyadenylation using a substrate that contains an AAUAAA but lacks a downstream element (SV40 + 5 substrate) and thus is not a substrate for cleavage. Therefore, although the processes of 3 ' cleavage and polyadenylation are normally tightly linked in vivo, they are distinct events that can be separated in vitro (Manley 1983; Hart et al. 1985; Moore and Sharp 1985; Zarkower et al. 1986). We conclude that the in vitro reaction does reflect the in vivo addition of the poly(A) tail and that the factors responsible for this reaction are present and active in the nuclear extract.

\section{Fractionation of HeLa cell nuclear extracts for poly(A) addition factors}

Although there have been numerous previous reports of simple poly(A) polymerase activities in mammalian cells that purify as single components (for review, see Nevins 1984), the activity catalyzing the sequence-specific 200-nucleotide addition appears to be complex. As shown in Figure 2A, fractionation of a nuclear extract by

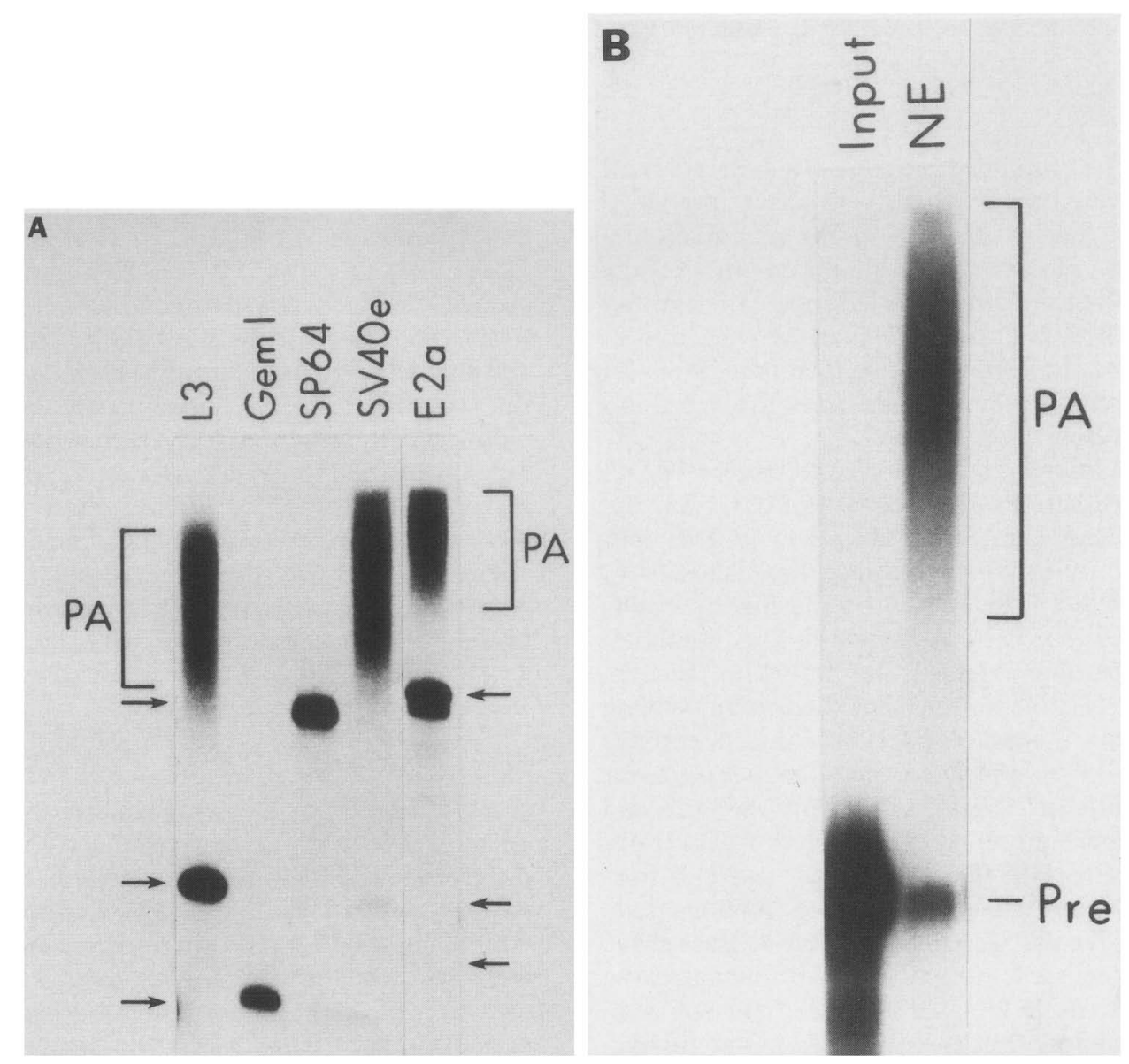

Figure 1. In vitro polyadenylation reflects the in vivo reaction. $(A)$ Various pre-mRNAs were incubated in a HeLa nuclear extract for $3 \mathrm{hr}$, and the products were purified and analyzed on a $5 \%$ polyacrylamide/urea gel. $(\rightarrow)$ The precursors; ([ ]) polyadenylated products. $(B)$ A transcript of the early SV40 poly(A) site truncated to +5 , which is not cleaved in the nuclear extract (see Fig. 1 of Gilmartin et al., this issue) was assayed in the poly(A) addition reaction. (Pre) precursor RNA. 

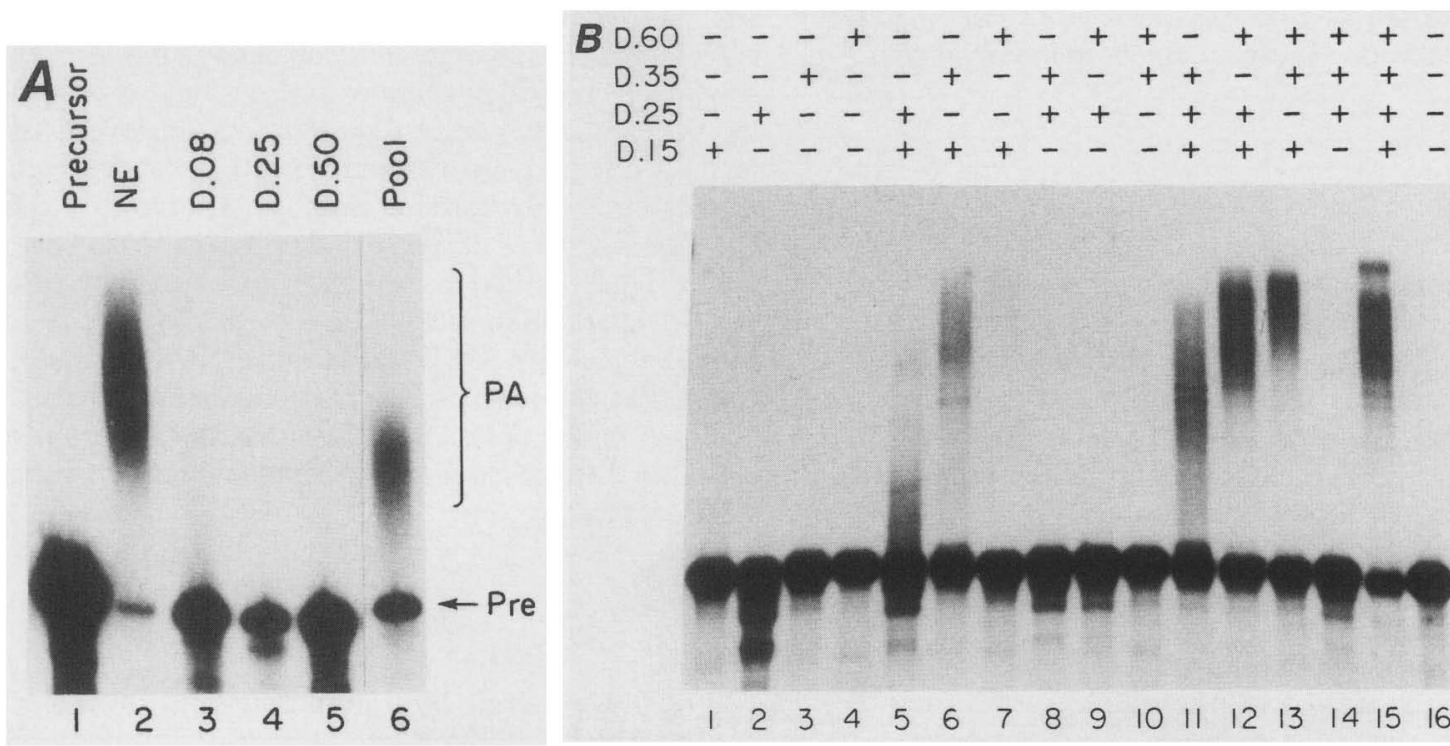

Figure 2. Fractionation of the nuclear extract by DEAE-Sephacel chromatography reveals multiple activities required for poly(A) addition. $(A)$ The nuclear extract was loaded and eluted from a DEAE-Sephacel column, as described in Methods. The flow-through fraction (D.08) and salt step elutions (D.25 and D.50) were concentrated and assayed independently or mixed together (Pool). (Lane 1) The L3 precursor without incubation; (lane 2) assay of the nuclear extract before fractionation. $(B)$ The nuclear extract was loaded onto a DEAE-Sephacel column and eluted, as described in Methods. The various step elutions were assayed alone or in combinations. (Lane 16) The L3 precursor RNA without incubation. The other lanes are assays of mixes of the DEAE $0.15,0.25,0.35$, and 0.60 molar step elutions for polyadenylation activity. The presence or absence of a fraction in the assay mix is indicated by a + or a - , respectively, above the lane. (PRE) The L3 precursor band; (PA) the polyadenylated products.

DEAE-Sephacel chromatography resulted in a set of fractions, no single one of which was active in poly(A) addition, as assayed by the shift of the precursor to a slower mobility. However, if equal amounts of the various DEAE fractions were pooled, near full activity was restored. Thus, we conclude that, as with the $3^{\prime}$ cleavage reaction, there are multiple factors required for the sequence-specific reaction that adds the $\sim 200$-nucleotide length of poly(A).

A more exhaustive fractionation was performed, resulting in DEAE fractions eluting at $0.15,0.25,0.35$, and $0.6 \mathrm{M} \mathrm{KCl}$. As shown in Figure $2 \mathrm{~B}$, assay of the individual fractions again revealed no activity (lanes 1-4), whereas activity was restored when the four fractions were recombined (lane 15). Assay of various combinations of the fractions yielded the following results. Omission of the D.15 fraction abolished activity (lane 14). Omission of the D.60 fraction reduced activity when the D.15, D.25, and D.35 fractions were present (lane 11) but abolished activity when only the D.15 and D.25 fractions were present (lane 5). Near full activity was obtained with only the D.15, D.25, and D.6 fractions (lane 12) or the D.15, D.35, and D.6 fractions (lane 13). From these results we conclude that at least three components are necessary for poly(A) addition, and these are contained in the D.15, D.25 or D.35, and D.6 fractions. It would appear that the middle component fractionates about equally between the 0.25 and $0.35 \mathrm{M}$ elutions and, thus, is found in both.

Because the D.25 fraction appeared to be equal to the D. 35 and because the D.35 fraction appeared to be par- tially contaminated with the D.6 activity, we have employed the D.15, D.25, and D.6 fractions for the subsequent assays of a reconstituted system. As shown in Figure 3A, assay of various RNA substrates demonstrated that the reconstituted system retained the specificity that was evident with the crude nuclear extract and that the size of the poly $(A)$ that was added was similar, based on electrophoretic mobility, to that added using the nuclear extract. Furthermore, as shown in Figure $3 \mathrm{~B}$, the polyadenylation in the reconstituted system required an AAUAAA and did not require cleavage of the substrate. Finally, the shift in migration of the precursor RNA was indeed due to the addition of poly(A), as shown by oligo(dT)-cellulose fractionation of the products (data not shown).

\section{One of the $\operatorname{poly}(A)$ addition factors is a nonspecific RNA component}

Many of the RNA processing reactions, including the 3'end cleavage reaction, require an activity with an essential RNA component (Hashimoto and Steitz 1986; Maniatis and Reed 1987; Sharp 1987; Gilmartin et al., this issue). The three fractions required for poly(A) addition were also tested for sensitivity to micrococcal nuclease digestion to determine whether any of the factors contained an RNA that was essential for activity. As shown in Figure 4A, both the D.15 and the D.25 fractions were resistant to micrococcal nuclease, whereas the D.6 activity was sensitive. Ryner and Manley (1987) have reported that micrococcal nuclease treatment of nuclear 
Figure 3. Characteristics of the poly $(\mathrm{A})$ addition reaction with the reconstituted system. $(A)$ Reconstituted column fractions add a proper size poly $(A)$ tail and require an authentic poly(A) site. In vitro polyadenylation reactions were carried out as described in Methods. Each reaction contained equal amounts of DEAE fractions D.15, D.25, and D.60 and employed the various indicated RNAs as substrates. $(\rightarrow)$ The position of the unprocessed precursor RNA. $(B)$ Poly $(\mathrm{A})$ addition in the reconstituted system requires an $A A U A A A$ and does not require cleavage. A transcript of the wild-type $\mathrm{L} 3$ poly(A) site (AAUAAA) or a transcript of a mutant L3 poly(A) site (AACAAA) was assayed in the reconstituted poly(A) addition reaction. Both transcripts have $3^{\prime}$ ends at the cleavage site (see Methods) and are not substrates for the cleavage reaction. extracts results in a nonspecific inhibition of 3 '-end processing activity because the simple readdition of nonspecific bulk RNA partially restores the inhibited reaction. Figure $4 \mathrm{~B}$ demonstrates that this is indeed the case for the D.6 fraction required for the poly(A) addition reaction. The addition of yeast RNA or Escherichia coli rRNA restores activity to a nuclease-treated fraction. These results suggest that RNA in the D. 6 fraction acts as a nonspecific competitor for RNA-binding activities that prevent efficient processing. The same conclusions were reached by Gilmartin et al. (this issue) concerning the function of the D.6 fraction in the 3 '-cleavage reaction, and we presume that the same function is provided by this material for both reactions. All subsequent assays have employed rRNA in conjunction with the D.15 and D.25 fractions.

\section{Relationship of activities involved in $\operatorname{poly}(A)$ addition and $3^{\prime}$-end cleavage}

Because the 3' cleavage of a pre-mRNA and the subsequent poly(A) addition are tightly coupled events in vivo, we have investigated the relationship of the activities involved in the two reactions.

The poly(A) site-processing reaction has been shown to be inhibited by antibodies with either U1 or Sm speci-

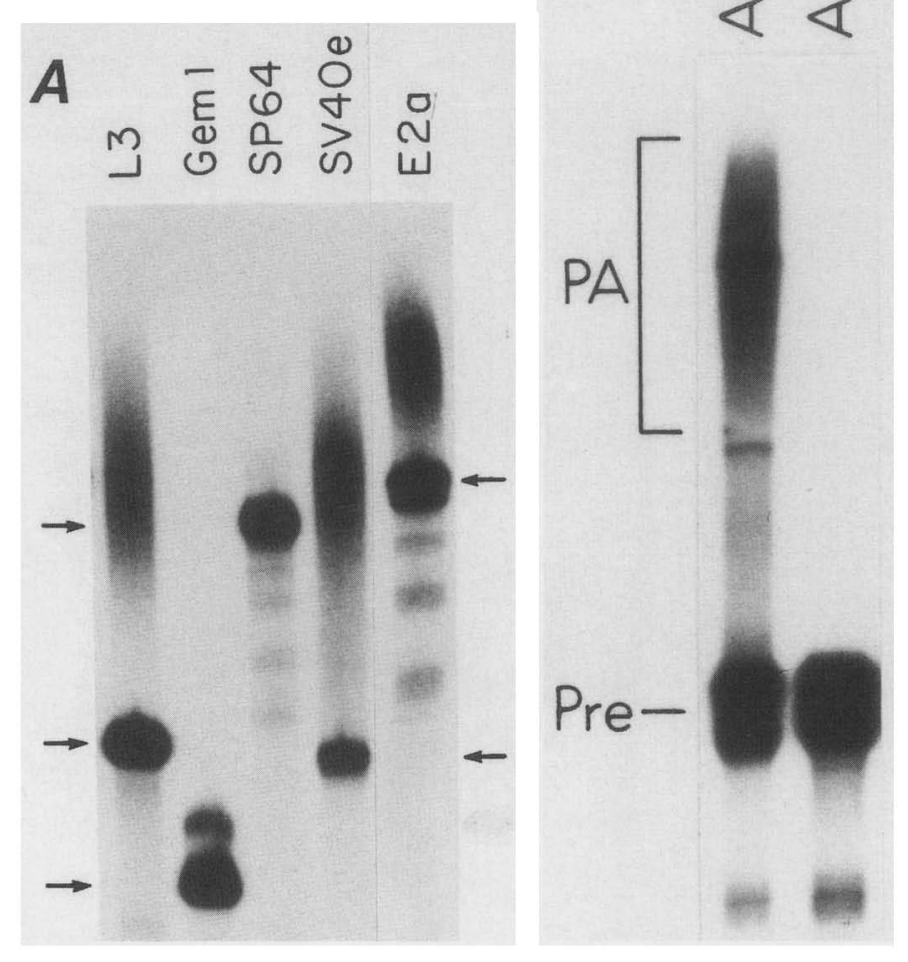

ficity (Moore and Sharp 1985; Sperry and Berget 1986). In each case, it was shown that normal 3' cleavage was inhibited, but it was not determined whether polyadenylation was also inhibited. In addition, Sm antisera immunoprecipitated complexes from polyadenylation reactions that were in association with the AAUAAA sequence element in the pre-mRNA (Hashimoto and Steitz 1986). And, as demonstrated by Gilmartin et al. (this issue), it appears that the D.25 fraction necessary for $3^{\prime}$ cleavage contains a small nuclear ribonucleoprotein particle (snRNP). However, as shown in Figure 4, the poly(A) addition reaction was not sensitive to micrococcal nuclease digestion. As a means of comparing the components of the two reactions further, we have tested the effect of various antisera on the in vitro 3 '-cleavage reaction and the poly(A) addition reaction, using the $\mathrm{L} 3$ precursor. Five different normal, nonlupus control sera had no effect either on the 3 '-cleavage reaction or the poly(A) addition reaction (data not shown). Inhibition by a Sm antiserum is illustrated in Figure 5A. In contrast to the inhibition of the $3^{\prime}$-cleavage reaction, the addition of the same antiserum $(\mathrm{Sm} \mathrm{1)}$, as well as another serum that also inhibited cleavage, had no effect on the poly|A) addition reaction (Fig. $5 \mathrm{~B}$ ). We thus conclude that the inhibition of the overall poly(A) site-processing reaction by anti-Sm sera is due to an inhibition of cleavage 

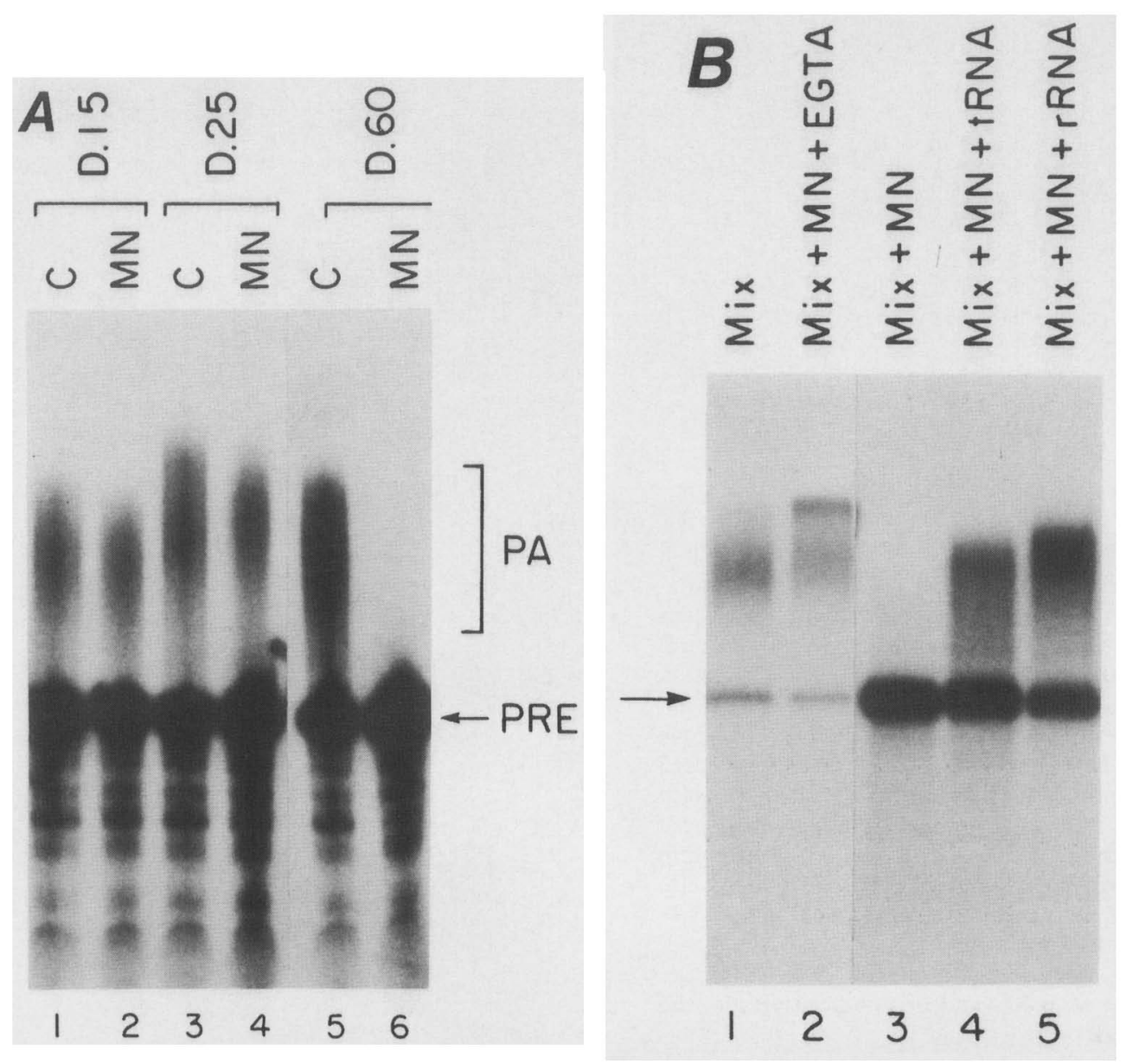

Figure 4. Effect of micrococcal nuclease on poly(A) addition activity. $(A)$ (MN) The indicated column fraction was treated with micrococcal nuclease $(90 \mathrm{U} / \mathrm{ml})$, as described in Methods, and then mixed with EGTA prior to the addition of L3 pre-mRNA and cofactors. Control reactions $(C)$ received EGTA prior to micrococcal nuclease. $(B)$ Nonspecific RNA restores activity to a micrococcal nuclease-treated D.60 fraction. The D.60 fraction was treated with micrococcal nuclease $(90 \mathrm{U} / \mathrm{ml})$ and mixed with EGTA, L3 pre-mRNA, and cofactors, including the D.25 and D.15 fractions (lane 3). (Lane 1) An assay with no micrococcal nuclease treatment; (lane 2) the EGTA was added prior to the micrococcal nuclease as a control; (lanes 4 and 5) assays where yeast RNA or E. coli rRNA $(1.5 \mu \mathrm{g})$ were added after micrococcal nuclease treatment of the D.60 fraction.

and that the cleavage activity possessing the Sm determinant, as well as the active RNA component, is not required for the poly(A) addition reaction.

\section{The D.15 activity has poly(A) polymerase activity}

In considering the possible roles of the activities involved in specific poly(A) addition, it was clear that one component must possess the enzymatic activity that polymerized AMP residues onto the $3^{\prime}$ end of the RNA substrate. Various studies have identified so-called poly(A) polymerase activities in mammalian cells (for review, see Edmonds and Winters 1976). For the purpose of this paper, we term the activity that polymerizes AMP onto an RNA 3' end without specificity as a poly(A) polymerase and the activities that reconstitute authentic and specific addition of an $~ 200$-nucleotide poly(A) tail as poly(A) addition. The poly(A) polymerase enzymes are generally characterized by their ability to add AMP residues to an RNA 3' terminus but without evidence for substrate specificity or formation of a physiological length of poly(A) tail. Indeed, the enzymes generally add only a few AMP residues to the RNA $3^{\prime}$ terminus. This lack of specificity might be due to the fact that such enzymes are completely unrelated to those that carry out the authentic poly(A) addition reaction, as assayed here or alternatively to the loss of specificity factors when these activities are purified. In this regard, it was of interest to note that the properties of the D.15 fraction necessary for poly $(\mathrm{A})$ addition were similar to a previously purified poly(A) polymerase from HeLa cells (Nevins and Joklik 1977). In particular, the HeLa poly(A) polymerase was predominantly nuclear, did not bind to a DEAE-Sephadex column, and sedimented slightly slower than bovine serum albumin (BSA). Each of these properties was consistent with the properties of the D.15 fraction. The assays for poly(A) addition activity with the separated fractions (e.g., Fig. 2) likely did not reveal 
Figure 5. Effect of Sm antibody on $3^{\prime}$ cleavage and poly(A) addition. Nuclear extract was preincubated with either a nonimmune control serum or autoimmune serum of Sm specificity, as described in Methods. L3 pre-mRNA and cofactors were then added, and the reaction proceeded in either the presence or absence of cordycepin triphosphate. The reactions were then processed and analyzed by polyacrylamide gel electrophoresis. (Left) An analysis of the 3'-cleavage reaction $1+$ cordycepin triphosphate); (right) the polyadenylation assay (- cordycepin triphosphate). The Sm 1 antiserum is the $\alpha-S m$ serum used in the cleavage assay on the left.
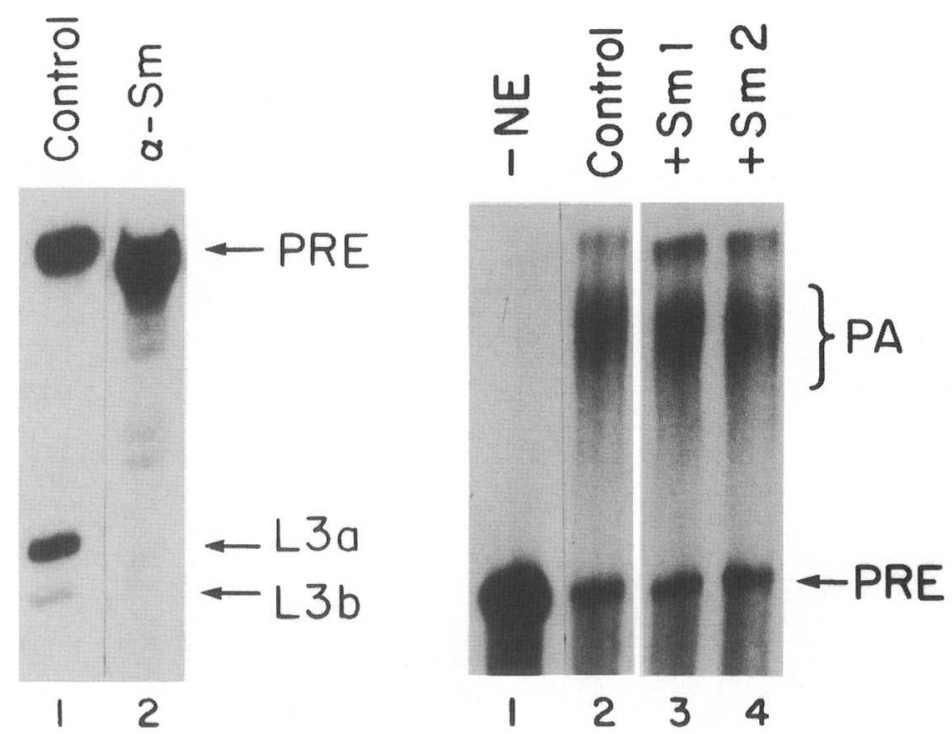

evidence of a poly(A) polymerase due to the short length of the tail added by these enzymes. To pursue the possible identity of the poly(A) polymerase and the D.15 fraction, we fractionated the D.15 activity further and assayed both for the reconstituted poly(A) addition activity, as well as for the ability to polymerize AMP in the absence of the D.25 fraction. The results of these analyses are shown in Figure 6. The D.15 fraction was first applied to a FPLC Mono $\mathrm{S}$ column and then eluted with a $75-500 \mathrm{mM} \mathrm{KCl}$ gradient. Assay of each fraction for ATP incorporation, in the absence of the D.25 fraction $[\operatorname{poly}(\mathrm{A})$ polymerase], and reconstituted poly(A) addition activity (in concert with the D.25 fraction) is shown in Figure 6A. As can be seen, both activities coeluted from the Mono S column, with the peak of activity between fractions 46 and 52 . The Mono $S$ activity was pooled, applied to a poly $(\mathrm{A})$-Sepharose column, and eluted with a $75 \mathrm{~mm}$ to $1 \mathrm{M} \mathrm{KCl}$ gradient. Again, both the simple poly(A) polymerase activity and the reconstituted poly(A) addition activity coeluted (Fig. 6B). Thus, both activities cofractionate through DEAE-Sephacel, Mono S, and poly(A)-Sepharose chromatography. In addition, further analysis by glycerol gradient sedimentation revealed both activities cosedimenting, consistent with a native molecular weight of $\sim 50,000$ (data not shown). These results therefore strongly suggest that the $\mathrm{D} .15$ fraction is a poly $(\mathrm{A})$ polymerase which, when as- sayed alone, lacks the specificity of the reconstituted reaction (see Fig. 2, lane 1).

\section{Discussion}

These studies demonstrate that multiple factors are required for the accurate and specific addition of a poly(A) tract to a mRNA $3^{\prime}$ terminus. Although it is not yet possible to say exactly how many components are involved in poly(A) addition, certainly there are at least two. An additional fraction (D.6) appears simply to sequester an inhibitor(s), probably RNA-binding proteins, and may well be dispensable once the other processing activities are further purified. The results of this study and that by Gilmartin et al. (this issue) therefore define a considerable complexity to the process of mRNA 3 '-end formation. The finding that multiple components are required for poly(A) addition was a somewhat surprising result as one could well imagine that a single enzyme could possess the appropriate enzymatic activity and substrate specificity to carry out the reaction. However, the requirement of multiple factors in the reaction suggests that the various events of recognition and polymerization may be separate.

The results presented in this paper suggest a role for one of the poly $(\mathrm{A})$ addition factors. We anticipated that the poly(A) addition process must involve a poly $(\mathrm{A})$ poly-

Figure 6. The D.15 fraction has poly(A) polymerase activity. (A) Mono S chromatography. The D.15 fraction was applied to a FPLC Mono S column in $75 \mathrm{mM} \mathrm{KCl}$. The column was washed with $75 \mathrm{mM} \mathrm{KCl}$ and then eluted with a gradient, ranging from 75 to $500 \mathrm{~mm}$ $\mathrm{KCl}$. Fractions were assayed for nonspecific poly(A) polymerase activity, as described in Methods, and for the reconstituted specific poly(A) addition activity, in conjunction with the D.25 fraction. The graph depicts the nonspecific poly(A) polymerase activity, and the reconstituted assays are shown in the inset. $(B)$ Poly $(A)-S e p h a r o s e$ chromatography. The Mono $S$ fractions containing activity (fractions 46-52) were pooled, dialyzed to $75 \mathrm{mM} \mathrm{KCl}$, and applied to a poly(A)-Sepharose column. The column was eluted with a 75 $\mathrm{mM}-1 \mathrm{M} \mathrm{KCl}$ gradient, and fractions were assayed for poly(A) polymerase activity and reconstituted poly $(\mathrm{A})$ addition activity. 
McDevitt et al.
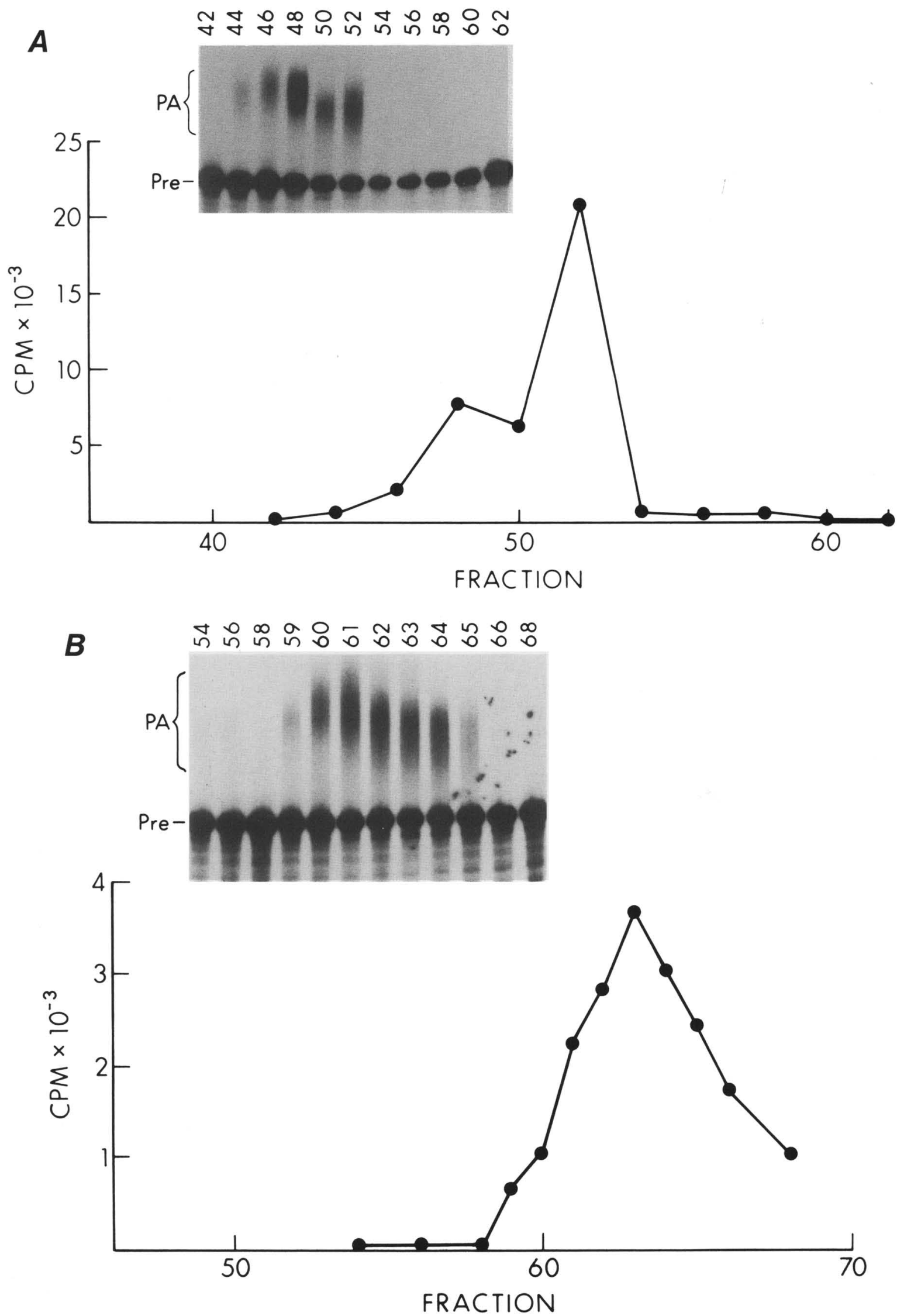
merase and possibly an additional factor that mediates recognition of the AAUAAA element. Previous work has identified a HeLa poly(A) polymerase activity, predominantly localized to the nucleus, with physical properties similar to the D.15 fraction identified here. The HeLa nuclear poly(A) polymerase did not bind to a DEAE matrix, it sedimented in a glycerol gradient slightly slower than BSA and, when purified to homogeneity, consisted of a single polypeptide of 50,000 molecular weight (Nevins and Joklik 1977). Additional purification of the D.15 activity suggests that, indeed, it may be the same poly(A) polymerase as the two activities copurify through three chromatographic steps. Although the ultimate proof of this identity will likely require an antibody that is capable of inhibiting both activities, the data presented here strongly suggest that the D. 15 component is the enzymatic activity that polymerizes AMP residues.

If the D.15 activity is a poly|A) polymerase, the function of the D. 25 fraction may be to provide specificity to the process such as sequence recognition and/or formation of the correctly sized 200-nucleotide poly(A) tract. Of interest is the recent observation of Wilusz and Shenk (1988) of a factor that interacts with RNA via a recognition of the AAUAAA element. This activity elutes from a DEAE column at $300-400 \mathrm{~mm} \mathrm{KCl}$ and thus may be equivalent to our D.25 activity, which was found equally in the 250 and $350 \mathrm{~mm}$ fractions. It is tempting to speculate that the D.15 fraction contains a poly(A) polymerase activity that, if assayed alone, does not have substrate specificity nor does it add a physiological length of poly(A). But, when combined with the D.25 fraction, which may be the AAUAAA-binding factor, specificity is imparted to the poly(A) polymerase. Of course, the final resolution must await the complete purification of these activities, but, clearly, the definition of individual components is an important step in that direction.

Given the tight coupling of $3^{\prime}$-end cleavage and polyadenylation in vivo, we presume that the various activities involved in these reactions are very likely components of a large complex that associates with the nascent pre-mRNA. Recent experiments employing native gel electrophoresis have begun to provide physical evidence for such complexes (Skolnik-David 1987; Zhang and Cole 1987). In addition, an analysis of complex formation involving the AAUAAA and the ability of certain pre-mRNAs to compete for complex formation suggested a common AAUAAA recognition in cleavage and poly(A) addition (Zarkower and Wickens 1987). Specifically, it was observed that a precursor truncated to the poly(A) site, which was functional in poly(A) addition but was not a cleavage substrate, could compete for the cleavage reaction. These investigators therefore concluded that the same complex probably was involved in both processes. Although our results do not provide any direct evidence for a common recognition factor for the two events, it remains a possibility that a shared component was not fractionated by our procedures. For in- stance, the AAUAAA recognition factor (Wilusz and Shenk 1988), which could well be the D.25 poly(A) addition activity identified in our studies, would also be present in the D. 25 cleavage fraction in addition to the presumed snRNP required for cleavage. The ultimate resolution of the roles of the various factors and their in vivo interactions to mediate poly(A) site formation will require the complete purification of the factors and the production of antibody reagents to serve as molecular probes for structure and function. Nevertheless, the characterization of the complexity, as described here, sets the stage for the next line of work.

\section{Methods}

Cells

HeLa cells were grown in suspension in Joklik's modified minimal essential medium (MEM), containing $5 \%$ calf serum.

\section{Preparation of nuclear extracts}

Nuclear extracts were prepared essentially as described by Dignam et al. (1983), except that nuclei were extracted with $350 \mathrm{mM} \mathrm{KCl}$ and all buffers contained $0.1 \mathrm{~mm}$ phenylmethylsulfonyl fluoride (PMSF).

\section{Column chromatography}

$D E A E-S e p h a c e l$ Procedures for fractionation of extracts on DEAE-Sephacel were as described by Gilmartin et al. (this issue).

Mono $S$ An aliquot of the D.15 fraction $(10 \mathrm{ml})$ was diluted in half in buffer A (Gilmartin et al., this issue) and applied to a $1-\mathrm{ml}$ FPLC Mono S column. The column was washed with 10 $\mathrm{ml}$ of starting buffer and eluted with a $15-\mathrm{ml}$ gradient $175-500$ $\mathrm{mM} \mathrm{KCl}$. Fractions of $0.5 \mathrm{ml}$ were collected.

Poly (A)-Sepharose Pooled Mono S fractions were dialyzed to $75 \mathrm{~mm} \mathrm{KCl}$ and applied to a $1-\mathrm{ml}$ poly $(\mathrm{A})$-Sepharose column. The column was washed with $10 \mathrm{ml}$ of starting buffer and then eluted with a $15-\mathrm{ml}$ gradient, ranging from $75 \mathrm{mM}$ to $1 \mathrm{M} \mathrm{KCl}$. Fractions of $0.5 \mathrm{ml}$ were collected.

\section{RNA precursors}

RNA precursors were synthesized as described previously (Hart et al. 1985), and stored in $30 \%$ ethanol at $-20^{\circ}$. The Geml precursor was obtained by transcription of pGem1 (Promega) linearized with HhaI. SP64 contains an 18-nucleotide insertion at the BamHI site and was linearized with HaeIII. The L3, SV40e, and E2A precursors are described by Gilmartin et al. (this issue). To prepare the L3 RNAs that terminate at the cleavage site, the $\mathrm{p} \Delta 7 \mathrm{C}$ plasmid (Gilmartin et al., this issue) was cleaved with $B a m H I$ and EcoRI to remove the L3 poly(A) site [BamHI cleaves $35 \mathrm{bp} 5^{\prime}$ to the AAUAAA, and EcoRI cleaves in the polylinker downstream of the poly(A) site]. Oligonucleotides of wild-type 
L3 poly(A) site sequence or sequence containing an AACAAA substitution were synthesized. Each oligonucleotide pair also contained an AccI site at the normal $3^{\prime}$-cleavage site (by changing the normal sequence of GTACAC to GTATAC). Each was cloned in the EcoRI and BamHI sites of $\mathrm{p} \triangle 7 \mathrm{C}$ and employed for RNA synthesis after linearization with AccI. The resulting RNAs possess a $3^{\prime}$ terminus that extends 1 nucleotide beyond the normal cleavage site.

\section{$\operatorname{Poly}(A)$ addition assay}

Reactions were carried out essentially as described by Hart et al. (1985), except $2 \%$ polyvinyl alcohol was included. For mixing experiments, $60 \%$ of the final reaction volume was composed of protein fractions containing $100 \mathrm{mM} \mathrm{KCl}, 0.2 \mathrm{mM}$ EDTA, 10\% glycerol, $20 \mathrm{mM}$ HEPES (pH 7.9), $0.5 \mathrm{mM}$ dithiothreitol (DTT), and $0.1 \mathrm{mM}$ PMSF. The $25-\mu \mathrm{l}$ reactions also included the following: $1 \mathrm{mM}$ ATP, $5 \mathrm{mM}$ creatine phosphate, 0.7 $\mathrm{mM} \mathrm{MgCl}_{2}, 2 \%$ polyvinyl alcohol, and $1.5 \mu \mathrm{g}$ rRNA E. coli (Pharmacia), where indicated.

\section{Micrococcal nuclease treatment}

Procedures for treating fractions with micrococcal nuclease were described by Gilmartin et al. (this issue).

\section{Antibody treatment}

Antisera were prepared as described by Hendrick et al. (1981). The specificity of the patient sera has been investigated thoroughly (Fisher et al. 1985; Reeves et al. 1986). Eleven microliters of nuclear extract was incubated with 3.5 microliters of buffer or antisera for $30 \mathrm{~min}$ on ice; the additional reactants including the RNA precursor were then added. After a 3-hr incubation, samples were processed and analyzed as above.

\section{$\operatorname{Poly}(A)$ polymerase assay}

Assays for poly(A) polymerase activity were performed as described previously (Nevins and Joklik 1977). The primer was 293-cell RNA, and HEPES buffer $(\mathrm{pH} 7.91$ was used instead of Tris.

\section{Acknowledgments}

We thank Suzanne Scarola for her excellent assistance in the preparation of nuclear extracts and Dr. Jan Lakota for contributing to the analyses shown in Figure 3B. M.A.M. is a National Institutes of Health (NIH) Medical Scientist Training Program Fellow. This work was supported by a grant from the NIH (GM35894).

\section{References}

Birnstiel, M.L., M. Busslinger, and K. Strub. 1985. Transcription termination and 3 ' processing: The end is in site! Cell 41: $349-359$.

Dignam, J.D., R.M. Lebovitz, and R.G. Roeder. 1983. Accurate transcription initiation by RNA polymerase II in a soluble extract from isolated mammalian nuclei. Nucleic Acids Res. 11: $1475-1489$.

Edmonds, M. and M.A. Winters. 1976. Polyadenylate polymerases. Prog. Nucleic Acid Res. Mol. Biol. 17: 149-179.

Fisher, D.E., W.E. Reeves, R. Wisniewolski, R.G. Lahita, and N. Chiorazzi. 1985. Temporal shifts from Sm to ribonucleoprotein reactivity in systemic lupus erythematosus. Arthritis Rheum. 28: 1348-1355.

Hart, R.P., M.A. McDevitt, and J.R. Nevins. 1985. Poly(A) site cleavage in a HeLa nuclear extract is dependent on downstream sequences. Cell 43: 677-683.

Hashimoto, C. and J.A. Steitz. 1986. A small nuclear ribonucleoprotein associates with the AAUAAA polyadenylation signal in vitro. Cell 45: 581-591.

Hendrick, J.P., S.L. Wolin, J. Rinke, M.R. Lerner, and J.A. Steitz. 1981. Ro small cytoplasmic ribonucleoprotein are a subclass of La ribonucleoproteins: Further characterization of the Ro and La small ribonucleoproteins from uninfected mammalian cells. Mol. Cell. Biol. 1: 1138-1149.

Maniatis, T. and R. Reed. 1987. The role of small nuclear ribonucleoprotein particles in pre-mRNA splicing. Nature 325: 673-678.

Manley, J.L. 1983. Accurate and specific polyadenylation of mRNA precursors in a soluble whole-cell lysate. Cell 33: 595-605.

Manley, J.L., P.A. Sharp, and M.L. Gefter. 1982. RNA synthesis in isolated nuclei: Processing of adenoviruses serotype 2 late messenger RNA precursors. J. Mol. Biol. 159: 581-599.

Manley, J.L., H. Yu, and L. Ryner. 1985. RNA sequence containing hexanucleotide AAUAAA directs efficient mRNA polyadenylation in vitro. Mol. Cell. Biol. 5: 373-379.

Moore, C.L. and P.A. Sharp. 1985. Accurate cleavage and polyadenylation of exogenous RNA substrate. Cell 41: 845-855.

Nevins, J.R. 1983. The pathway of eukaryotic mRNA formation. Annu. Rev. Biochem. 52: 441-466.

- 1984. The role of poly(A) in mRNA biogenesis. In Processing of RNA (ed. D. Apirion), CRC Press, Boca Raton, Florida.

Nevins, J.R. and J.E. Darnell. 1978. Steps in the processing of Ad2 mRNA: Poly(A) nuclear sequences are conserved and poly A addition precedes splicing. Cell 15: 1477-1493.

Nevins, J.R. and W.K. Joklik. 1977. Isolation and partial characterization of the poly(A) polymerases from HeLa cells infected with vaccinia virus. J. Biol. Chem. 252: 6939-6947.

Reeves, W.H., D.E. Fisher, R. Wisniewolski, A.B. Gottlieb, and N. Chiorazzi. 1986. Psoriasis and Raynaud's phenomenon associated with autoantibodies to $\mathrm{U} 1$ and $\mathrm{U} 2$ small nuclear ribonucleoproteins. N. Engl. J. Med. 315: 105-111.

Ryner, L.C. and J.L. Manley. 1987. Requirements for accurate and efficient mRNA $3^{\prime}$ end cleavage and polyadenylation of a simian virus 40 early pre-RNA in vitro. Mol. Cell. Biol. 7: 495-503.

Sharp, P.A. 1987. Splicing of messenger RNA precursors. Science 235: 766-771.

Skolnik-David, H., C.L. Moore, and P.A. Sharp. 1987. Electrophoretic separation of polyadenylation-specific complexes. Genes Dev. 1: 672-682.

Sperry, A.O. and S.M. Berget. 1986. In vitro cleavage of the simian virus 40 early polyadenylation site adjacent to a required downstream TG sequence. Mol. Cell. Biol. 6: 47344741 .

Wilusz, J. and T. Shenk. 1988. A $64 \mathrm{kD}$ nuclear protein binds to RNA sequences that include the AAUAAA polyadenylation motif. Cell (in press).

Zarkower, D. and M. Wickens. 1987. Formation of mRNA 3' 
termini: Stability and dissociation of a complex involving the AAUAAA sequence. EMBO J. 6: 177-186.

Zarkower, D., R. Stephenson, M. Sheets, and M. Wickens. 1986. The AAUAAA sequence is required both for cleavage and for polyadenylation of simian virus 40 pre-mRNA in vitro. Mol. Cell. Biol. 6: 2317-2323.

Zhang, F. and C.N. Cole. 1987. Identification of a complex associated with processing and polyadenylation in vitro of herpes-simplex virus type I thymidine kinase precursor RNA. Mol. Cell. Biol. 7: 3277-3286. 


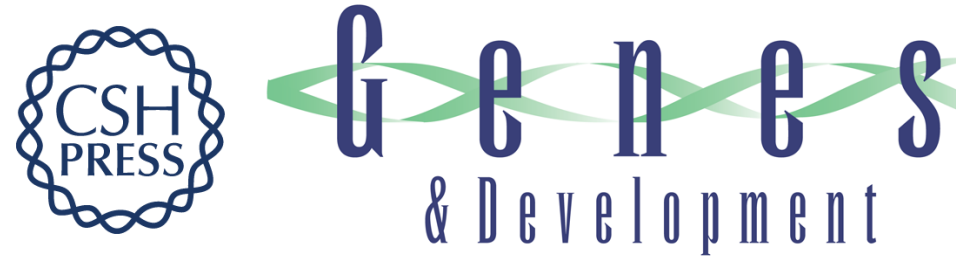

\section{Multiple factors are required for poly(A) addition to a mRNA 3' end.}

M A McDevitt, G M Gilmartin, W H Reeves, et al.

Genes Dev. 1988, 2:

Access the most recent version at doi:10.1101/gad.2.5.588

$\begin{array}{ll}\text { References } & \begin{array}{l}\text { This article cites } 23 \text { articles, } 9 \text { of which can be accessed free at: } \\ \text { http://genesdev.cshlp.org/content/2/5/588.full.html\#ref-list-1 }\end{array}\end{array}$

License

Email Alerting Receive free email alerts when new articles cite this article - sign up in the box at the top Service right corner of the article or click here.

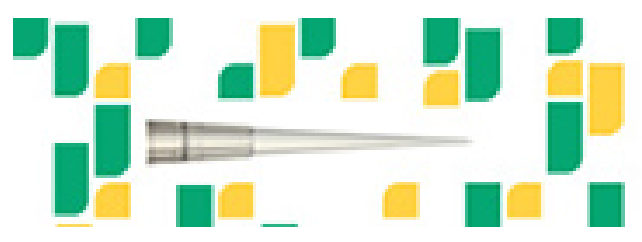

Focused on your science. 